\title{
Muséologies
}

Les cahiers d'études supérieures

muséologies

\section{Michael Blum}

\section{Marie Lavorel}

Volume 7, numéro 2, 2015

La collection muséale

URI : https://id.erudit.org/iderudit/1030255ar

DOI : https://doi.org/10.7202/1030255ar

Aller au sommaire du numéro

\section{Éditeur(s)}

Association Québécoise de Promotion des Recherches Étudiantes en Muséologie (AQPREM)

\section{ISSN}

1718-5181 (imprimé)

1929-7815 (numérique)

Découvrir la revue

Citer ce document

Lavorel, M. (2015). Michael Blum. Muséologies, 7(2), 153-161.

https://doi.org/10.7202/1030255ar

\section{Résumé de l'article}

Sa dernière exposition, intitulée Notre histoire, qui a eu lieu à la Galerie de l'Université du Québec à Montréal, prolonge ce travail de provocation d'un jugement critique où il s'approprie cette fois la forme muséale. Sur le mode de la parodie, il interroge le rapport à l'histoire et à la mémoire de la société québécoise et canadienne, tout en mettant le visiteur de cette exposition face au pouvoir idéologique du musée. Notre article se divise en deux parties. Dans un premier temps, nous laisserons la parole à l'artiste - sous la forme d'un entretien -, qui reviendra sur la genèse de ce projet, sur son processus et le contexte dans lequel il a réalisé cette exposition. Puis, dans un second temps, nous présenterons un bref compte rendu critique de cette exposition en nous intéressant particulièrement à l'utilisation de cette forme muséale et aux enjeux idéologiques et critiques qui la traversent.
Tous droits réservés (C) Association Québécoise de Promotion des Recherches Étudiantes en Muséologie (AQPREM), 2015
Ce document est protégé par la loi sur le droit d'auteur. L'utilisation des services d'Érudit (y compris la reproduction) est assujettie à sa politique d'utilisation que vous pouvez consulter en ligne.

https://apropos.erudit.org/fr/usagers/politique-dutilisation/ 
Entrevue un

\section{Michael Blum}

Marie Lavorel 


\section{L'œuvre de Michael Blum - artiste né à Jérusalem en} 1966 et installé à Montréal depuis quatre ans - est traversée par une relecture critique de l'histoire et de la culture moderne et contemporaine. Par le biais d'installations, de publications et de vidéos, il interroge les rouages idéologiques, politiques, économiques et médiatiques des multiples sociétés qu'il habite. Que ce soit notamment avec les projets A Tribute to Safiye Behar (2005), Cape TownStockholm (2007), Faktories und Felder (2012), Monument to the Birth of the 20th Century (2005) ou Guerre et paix (2014), il crée une ouvre polymorphe aux récits parodiques, documentaires et polyphoniques qui construit une réflexion critique sur l'écriture de l'histoire, de la mémoire et de l'oubli.

Sa dernière exposition, intitulée Notre histoire, qui a eu lieu à la Galerie de l'Université du Québec à Montréal, prolonge ce travail de provocation d'un jugement critique où il s'approprie cette fois la forme muséale. Sur le mode de la parodie, il interroge le rapport à l'histoire et à la mémoire de la société québécoise et canadienne, tout en mettant le visiteur de cette exposition face au pouvoir idéologique du musée. Notre article se divise en deux parties. Dans un premier temps, nous laisserons la parole à l'artiste - sous la forme d'un entretien -, qui reviendra sur la genèse de ce projet, sur son processus et le contexte dans lequel il a réalisé cette exposition. Puis, dans un second temps, nous présenterons un bref compte rendu critique de cette exposition en nous intéressant particulièrement à l'utilisation de cette forme muséale et aux enjeux idéologiques et critiques qui la traversent. 
Marie Lavorel - Bonjour Michael, j'aimerais revenir sur la genèse de votre projet d'exposition Notre histoire qui a eu lieu à la Galerie de l'UQAM du 2 septembre au 4 octobre 2014. Quel est le point de départ de ce projet, de cette réflexion sur les identités québécoise et canadienne?

Michael Blum - Le point de départ, c'est une invitation de la Galerie de l'UQAM, une carte blanche, qui m'a amené à retourner à la racine des choses. La première fois que je suis venu à Montréal, c'était en 1992. J'avais été frappé par cette bipolarité de Montréal, où tout est dédoublé, et ce n'est pas que le bilinguisme, ça va beaucoup plus loin, on pourrait qualifier cette relation de consubstantialité conflictuelle. J'avais pris des notes là-dessus et ensuite j'étais reparti. Mais en revenant, en 2010, cela m'a frappé de nouveau. J'ai aussi une obsession du langage, j'ai passé toute ma vie entre deux ou trois langues, entre des appartenances ethno-nationales multiples (et non exclusives), donc c'est un sujet auquel je suis très sensible. À Montréal, c'est fossilisé dans les institutions, toute la vie est organisée autour de cela et en même temps c'est une espèce de tabou.

Mes projets les plus pertinents en termes d'écho culturel ont toujours eu lieu dans des endroits où j'étais étranger, où j'avais un regard que seul un étranger pouvait se permettre sur un sujet controversé ou tabou, ou du moins délicat, que ce soit Atatürk en Turquie, le nazisme en Autriche, ou la dialectique franco-anglo à Montréal. C'était clair que j'allais faire ce genre de projet un jour.

Très tôt dans le développement du projet s'est imposée l'idée d'ériger un double musée national. Deux musées parallèles qui raconteraient la même histoire de deux points de vue différents. Cette structure était là dès le début. C’est clair que c'est un projet qui, au Québec, ne pouvait se réaliser que sur invitation.

M.L. - Vous avez eu une grande marge de manœuvre avec cette carte blanche...

M.B. - Vous avez raison de le souligner, car ce n'est pas toujours le cas! Et une galerie universitaire est l'institution idéale pour un tel projet, car elle échappe aux pressions néolibérales et conservatrices du conseil d'administration du musée et à la frilosité inhérente aux centres d'artistes.

M.L. - Vous avez développé un processus créatif fondé sur une enquête par sondage. Votre idée, votre forme d'exposition était là au départ, mais ensuite vous êtes passé rapidement à cette idée de sondage. Pourquoi avez-vous utilisé ce moyen? Est-ce par ce biais que vous validiez les propos que vous alliez tenir dans l'exposition? Est-ce que cela vous permettait également d'être encadré et de questionner ces notions d'identité avec un public choisi? 
M.B. - C'était tout ça en même temps, de façon assez organique. Mais il m'a paru clair qu'il fallait aussi la voix des autres. C'était effectivement assez contradictoire. Je voulais d'un côté faire entendre ma voix sur cette question - d'autant plus que les étrangers qui vivent au Québec se sentent rarement libres d'en parler -, mais, en même temps, je ne voulais pas que ce soit une voix dans le désert. Il fallait faire participer une multiplicité de voix. Il s'agissait par ailleurs de baliser et de déminer le champ dans lequel je m'apprêtais à intervenir. Certaines de ces voix singulières m'ont éclairé sur des aspects particuliers par des formulations spécifiques. Par exemple, Louise Vigneault : «Le Québec est une culture qui cherche un pays et le Canada est un pays qui cherche sa culture. " J'avais bien compris la situation pour ce qui est du Québec, mais ça m'a aidé à prendre la mesure de la réécriture de l'histoire opérée par le gouvernement de Stephen Harper. Comment il s'approprie l'idée du Nord! Et 1812! C'est hallucinant, cette façon d'écrire une histoire pour fabriquer de l'identité nationale sur demande ! Du coup, cela m'a encouragé à forcer un peu le trait dans mon musée canadien, d'où, par exemple, la liste des sponsors en prise beaucoup plus directe sur l'actualité que du côté québécois : 1812 Inc., Jews for Jesus, Circle of Canadian Creationists... J'aime aussi beaucoup la définition dialectique du Québec par Cheryl Sim: "An underdog and a bully at the same time."

M.L. - Les réponses que vous avez eues au sondage vous ont-elles permis, même si vous ne vous en êtes pas inspiré pour produire les dispositifs de l'exposition, de tester certaines sensibilités, les limites jusqu'où vous pouviez aller dans votre discours?

M.B. - Assurément, même si je pense que les limites ne sont pas en termes de ce dont on parle, mais plutôt de comment on en parle et du ton qu'on emploie. J'ai surtout travaillé à trouver le ton juste, mais en termes de sujet, je voulais tout aborder, tout dire - c'était justement là l'essence du projet! Dans la vie quotidienne, on passe son temps à ne rien dire puisque c'est un sujet socialement accepté comme tabou dans l'intérêt commun. Là, je voulais au contraire créer un espace où la parole était libre. Et c'est aussi pour cela que j'ai choisi un ton aussi satirique, pour bien marquer que l'on est dans un espace différent, où tout peut se dire. Et l'effet cathartique est à la mesure du tabou brisé... Ce qui symbolise cela, par exemple, c'est le mur des grandes réalisations. Les grandes réalisation [sic], je l'ai écrit d'abord sans faute d'orthographe. C'était une forme d'autocensure puisque j'aurais voulu mettre des fautes d'orthographe partout, mais j'avais peur d'insulter les gens, alors que je cherche au contraire à séduire le visiteur, à endormir sa vigilance et ses éventuels préjugés négatifs. Mais j’ai observé les réactions de l'équipe de la Galerie et j'ai vite compris que j'étais trop timoré. J'ai donc finalement remis réalisation au singulier! C'est un mouvement de va-etvient, une négociation permanente entre la liberté et la responsabilité. Mais au tout début de l'envoi du sondage, je tâtais le terrain, et je tâtonnais surtout. 
M.L. - Vous avez envoyé ce sondage autour de vous, uniquement au milieu universitaire et artistique?

M.B. - Non, j'ai écrit à beaucoup plus de gens, mais seuls la communauté universitaire et les producteurs culturels ont répondu. Même parmi mes proches, beaucoup n'ont pas répondu, jugeant l'exercice trop difficile, trop problématique ou même " inacceptable ». Les questions étaient très chaudes et il y avait beaucoup de coups à prendre, j'en conviens! Pour mes répondants aussi, il fallait trouver le ton juste...

J'ai eu accès au listing de presse de l'UQAM, ce qui m'a permis de contacter tous les journalistes et les éditorialistes au Québec et ailleurs au Canada. Pas un n'a répondu! J'ai contacté tous les députés fédéraux et provinciaux. Là non plus, aucune réponse, mais c'est dans l'ordre des choses. Le résultat est très montréalais, universitaire, mais pas exclusivement. J'étais quand même content d'avoir quelques personnes en dehors. Mais ce qui m’a poussé à faire un livre, est la grande qualité des réponses. Je m'attendais à beaucoup de réponses tranchées, voire caricaturales, mais en fait la position de chacun est beaucoup plus nuancée que l'image que l'on cherche à donner publiquement.

Il y a eu aussi la question des élections, le "timing " de la vie politique au Québec allait plus vite que celui de l'exposition. Je travaille toujours avec des histoires plus lentes, qui viennent du passé et qui se prolongent encore dans le présent, et là, tous ces temps différents se superposaient, du temps volatile très court de la vie politique d'aujourd'hui au temps long braudélien du ressentiment et du mépris.

M.L. - Avez-vous eu le besoin d'accompagner ce processus de lectures sur l'histoire québécoise et canadienne?

M.B. - J'ai commencé cela bien avant ce projet, même un peu avant d'arriver au Québec. Les mois qui ont précédé mon arrivée, je lisais les nouvelles locales et l'histoire du Québec, et parcourais les rues de Montréal sur Google Street View à la recherche d'un appartement! J'ai continué à lire sur la question - et je continue encore aujourd'hui -, parce que c'est un sujet fascinant avec une bibliographie infinie. Au printemps dernier, je lisais encore Mordecai Richler Oh Canada! Oh Québec!, la voix du polémiste pour me donner du courage! Mais là aussi c'était davantage une histoire de ton; l'histoire, je la connaissais déjà, ou les connaissais déjà, puisqu'on a deux versions aussi antinomiques qu'officielles de l'histoire... Au fond, c'est Montréal qui m'intéresse, le lieu où ces deux histoires se rencontrent, où la dialectique entre en jeu. Toute ma recherche, c'était en fait les quatre années passées à Montréal avant mon exposition. Une recherche intuitive, empirique et de tous les instants. 
M.L. - Le recours à la forme muséale n'a-t-il pas évacué quelque peu le propos sur l'identité développé au sein de votre exposition?

M.B. - Les concepts de musée et d'État-nation moderne sont nés en même temps et les deux sont inséparables. Pas de nation sans musée, pas de musée sans nation. Et puis, il me fallait un dispositif qui me permette de ne pas être frontal dans ma critique, de détourner l'attention. Utiliser un musée me permettait de dire par procuration toutes ces choses que je ne pouvais pas énoncer en personne.

M.L. - Vous êtes professeur à l'UQAM et vous exposez au sein de la Galerie de cette université. Est-ce que l'autorité de l'institution a parfois été une contrainte, une limite? En termes de réception, cela a-t-il posé problème?

M.B. - Bien au contraire. Je pense que mon statut de professeur m'a donné une légitimité universitaire cruciale pour mettre les répondants au sondage en confiance, et j'aurais probablement eu moins de réponses de collègues de l'UQAM et des autres universités si je n'avais été que artiste. Ayant été indépendant toute ma vie jusqu'en 2010, je suis très sensible à cette question de la légitimité institutionnelle. Mais, surtout, mon statut de professeur m'a permis de faire financer le projet par l'université, ce qui garantissait cette liberté de parole.

M.L. - Je voudrais revenir sur la visite guidée qui a été organisée par la Galerie. Lors de cette visite, certains participants ont parlé de fiction, d'" invraisemblable " à propos de votre travail, tandis que vous aviez expliqué que votre intention était de construire un récit entre réalités et clichés et que vous ne placiez pas ce projet du côté de la fiction. Qu'est-ce que cela révèle quant à la compréhension de votre démarche et de votre exposition? Est-ce qu'il y a une forme de déni?

M.B. - Je ne sais pas si c'est une forme de déni, mais en tout cas une façon de simplifier quelque chose de complexe, de définir l'indéfini et d'assurer que cela restera sans conséquence dans le monde réel. Je préfère le terme de parafiction, développé par Carrie Lambert-Beatty ${ }^{1}$ justement pour combler cette lacune, qui définit ces pratiques ambiguës qui utilisent la fiction, mais dans la sphère du réel, qui influencent le réel.

M.L. - Est-ce que ce projet a changé votre vision sur l'identité québécoise et canadienne? Et est-ce que cela a modifié votre statut d'immigré, votre façon d'habiter ici? 
M.B. - Le projet a surtout établi la preuve de ma bonne assimilation! J'emploie à dessein ce terme honni au Québec; or, dans ma culture familiale de juif cosmopolite européen, l'assimilation est une nécessité de survie, pas la menace de l'extinction. Mais ça, c'est une autre histoire...

\section{$\stackrel{*}{*}$}

L'exposition Notre histoire se présente sous la forme de deux musées: d'un côté un musée de l'histoire du Québec, de l'autre du Canada. Deux dispositifs muséaux distincts et autonomes sont ainsi proposés aux spectateurs de l'exposition. À gauche, on entre dans le musée consacré au Québec dont le texte d'introduction promet de révéler enfin la vraie histoire. Dès cette formule, le ton de l'exposition est posé, c'est sur le mode de la parodie que cette histoire sera contée. S'ensuit un parcours offrant au regard des objets sélectionnés par l'artiste parmi ceux de la collection de la Galerie de l'UQAM qu'il transforme en objets témoins de son histoire québécoise et qu'il alimente par la présence de textes et de cartels longs. Une roche sacrée symbole de la québécitude, un crâne d'un Iroquoien, un cor ancien, un vieux bréviaire et une boîte à pilules sont, parmi d'autres objets, les supports détournés et inventés du récit d'une histoire québécoise parodiée. Des origines avec la roche sacrée à l'histoire contemporaine formalisée par un mur de glorieuses réalisations québécoises où se côtoient entres autres le pont Champlain, les tempêtes de neige, les libéraux ou la république de la Nouvelle-France, Blum construit une histoire du Québec qui provoque un rire franc et en même temps une réflexion critique tant sur l'écriture de l'histoire que sur l'autorité muséale. Le visiteur est dans un espace modelé par une muséologie centrée sur l'objet, utilisant vitrines, cartels et textes introductifs. Visuellement, l'illusion muséale fonctionne à tel point que certains visiteurs cherchent à vérifier s'il s'agit d'objets authentiques et fondateurs de l'histoire québécoise. Et pourtant le cartel adjacent, le choix de ces objets saugrenus, les textes truffés d'anachronismes et d'événements historiques aux noms transformés (la bataille des plaines d'Abraham devient par exemple la bataille de Moïse) : tout concourt à afficher ce ton parodique, à rappeler au visiteur qu'on est en train de lui raconter des histoires. Mais l'utilisation maîtrisée de la forme muséale par Blum place d'emblée le regardeur dans un dispositif lié à l'authenticité, à la transmission d'un savoir autorisé, vérifié, validé.

La parodie n'en est que plus efficace. Elle se produit à deux niveaux: une parodie du musée et une parodie de l'histoire. Blum imite ${ }^{2}$ la forme muséale, tout en s'en moquant par la création d'un contenu où la parodie prend tout son sens. Le discours du musée, du côté québécois, est ainsi transformé en un conte mythique supporté par des expôts, des cartels et des textes. Plus on avance dans ce parcours, plus cette autorité muséale 
détournée laisse la place à un espace en friche correspondant à l'histoire moderne et contemporaine du Québec. Un musée inachevé, dont l'exposition est en montage, par manque de financement, de volonté politique ou de contenu. Dès lors, d'une imitation d'un espace muséal classique dont le contenu est cependant parodié, on passe à un espace formel plus près d'une installation d'art contemporain. L'artiste s'amuse avec les codes muséaux sur un ton burlesque et construit un univers fantasmé. La place réservée au gardien de musée, qui, selon les mots de l'artiste, aurait pu être habitée par une personne assise fumant continuellement, en est un bel exemple.

Du côté du musée canadien, on assiste à une exposition qui se déploie sous la forme d'un musée d'histoire nationale imposant, autoritaire, symbole d'une identité forte, affirmée avec fierté. Le ton parodique est tout autant présent, mais est distillé au sein d'un espace dédié à la puissance d'une nation. Si les objets choisis représentant les grandes inventions canadiennes sont définitivement ridicules, le dispositif de présentation et la scénographie dans son ensemble maintiennent l'illusion de puissance. Une nouvelle fois, l'artiste démontre sa maîtrise de la forme muséale tout en véhiculant une réflexion critique sur la teneur idéologique des discours historiques construits par les musées.

En effet, au-delà de la construction d'un récit historique québécois et canadien utilisant les ressorts de la parodie définie par Gérard Genette ${ }^{3}$, l'artiste propose un récit qui provoque une réflexion sur l'écriture de l'histoire au Québec et au Canada. Le régime ludique, auquel la parodie appartient, décliné tout au long de l'exposition, permet à Blum de se distancier d'une intention agressive ou moqueuse vis-à-vis d'un sujet délicat, les identités historiques québécoise et canadienne. Mais il donne également la possibilité à l'artiste d'interroger sans heurter les sensibilités des visiteurs un régime historial sur lequel se fondent ces deux identités. Celui qu'il propose, bien que détourné et parodié, repose sur une écriture de l'histoire du Québec inachevée, en construction, face à une histoire du Canada à l'apparence maîtrisée, véhicule d'un discours nationaliste fort.

Au sein du musée québécois, il crée un nouveau récit collectif composé de multiples mythistoires qui reprennent les quatre piliers narratifs définis par Jocelyn Letourneau qui fonderaient actuellement la construction identitaire québécoise: la survivance, la quête de Soi, le destin dévié, la faute à l'Autre ${ }^{4}$.

3 Nous utilisons le terme parodie en référence à celui défini par Gérard GENETTE dans Palimpsestes: La littérature au second degré. Paris: Seuil, coll. « Points ", 1982.
4 LÉTOURnEAU, Jocelyn. «Mythistoires de Losers, introduction au roman historial des Québécois d'héritage canadien-français ". Histoire sociale / Social History, vol. 39, $\mathrm{n}^{\mathrm{o}} 77,2006$, p. 157-180, à la p. 165. 
Au sein du musée canadien, il met l'accent sur la capacité du dispositif muséal à instrumentaliser l'histoire au service d'un pouvoir politique en place ${ }^{5}$.

À travers Notre histoire, Michael Blum fait de l'espace muséal et de l'écriture de l'histoire un objet d'investigation qui amène à déconstruire l'idéologie sous-jacente à toute muséologie et offre au visiteur la possibilité de poser un regard critique sur ce que communique un musée d'histoire. Sa démarche éminemment réflexive rappelle que l'écriture de l'histoire au musée est avant tout une construction qui fonctionne sur des relations de pouvoir et qui dévoile une vision partielle et souvent partiale de la réalité. Dans ces temps contemporains où diverses positions idéologiques antagonistes s'affrontent violemment et où les analyses que l'on en fait sont souvent très réductrices et composées d'opinions médiatisées à outrance, il est salutaire d'adopter ce regard distant et critique sur ce qui fonde nos propres identités et d'interroger les dispositifs de communication qui les représentent.

5 Sur les liens entre pouvoir politique et milieu muséal, voir l'article de BERGERON, Yves. « Les liaisons dangereuses ou les relations troubles entre le politique et les musées canadiens ». THEMA: La revue des Musées de la civilisation, $\mathrm{n}^{\circ} 1,2014, \mathrm{p} .127-140$. Il y décrit notamment les politiques de commémorations mises en place par Stephen Harper et la nouvelle orientation du Musée canadien des civilisations devenu Musée canadien de l'histoire. Cette nouvelle appellation fait partie d'une politique plus large de redéfinition de ce que doit être l'identité canadienne selon le gouvernement conservateur qui se sert, pour véhiculer ces nouveaux discours identitaires, des institutions patrimoniales dont il assure la gouvernance. À ce propos, Blum a bien saisi l'importance du Musée dans la construction d'une Nation qui, à côté de la carte et du recensement, participe à l'édification d'un imaginaire national. (ANDERSON, Benedict. L'imaginaire national. Paris: La Découverte, 1996.) 